\title{
Molecular mechanisms of a traditional Chinese medicine in the treatment of dysfunctional uterine bleeding
}

Bo LV

Hunan Normal University

Yuan-de Peng

Chinese Academy of Agricultural Sciences

Wen-hui Fu

Hunan Agriculture University

Wen-jing Li

Hunan Agriculture University

Li-jun Chen ( $\nabla$ 19866408@qq.com )

Hunan Normal University

Zhi Wang

Hunan Normal University

\section{Research Article}

Keywords: Dysfunctional uterine bleeding, Xuebeng recipe, Endometrial hyperplasia, Hormone, mRNA, Regulation

Posted Date: April 27th, 2021

DOl: https://doi.org/10.21203/rs.3.rs-442423/v1

License: (c) (i) This work is licensed under a Creative Commons Attribution 4.0 International License. Read Full License 


\section{Abstract}

Dysfunctional uterine bleeding (DUB) is a common gynecological disease regulated by multiple hormones, which seriously affects female physical and mental health. Some drugs have been prompted to cure this disease, but most drugs have certain side effects and limitations. In the present study, we demonstrated an unexploited Chinese traditional medicine, a combination of Saururus chinensis, Celosia cristata and Spatholobus suberectus (Referred to as XBR), was capable of exerting hemostatic, coagulation and anti-inflammatory effects on SD rats. Furthermore, the XBR decreased the value of erythrocyte, hemoglobin, and hematocrit in serum for lowering blood stasis caused by DUB. Further studies were conducted to provide direct evidence that the recovery of endometrial hyperplasia was associated with the modulation of the hormones (follicle-stimulating hormone, luteinizing hormone, estradiol and progesterone). Besides, XBR altered mRNA expression levels of matrix metalloproteinase-1 and tissue inhibitor of matrix metalloproteinase- 1 on the uterine endometrium, thereby promoting the repair of proliferating endometrium in the rats. Collectively, our study indicates that XBR harbors a profoundly curative effect on the treatment of DUB, and explains the mechanism at the molecular and genes expression levels.

\section{Introduction}

As one of the oldest healing methods, traditional Chinese medicine (TCM), including herbal medicine, acupuncture, moxibustion (Tang et al., 2008), has been profoundly promoted and used in treating congestion, diabetes and malaria for a long time (Gan et al., 2010; Li et al., 2018; Xiao and Luo, 2018). Furthermore, accumulating evidence indicates that TCM-related therapy is able to improve spasticity (Yang et al., 2018), neurological function deficit (He, 2015) and mental status (Bai et al., 2015) effectively. Acupuncture can induce the release of corticotrophin-releasing factor and adrenocorticotropic hormone thus decrease sympathetic tonus (Eisenhardt and Fleckenstein, 2016). Artemisinin, honored in the context of the Nobel Prize winners 2015, was first isolated and tested to fight against malaria by Chinese researchers (Tu, 2016). Nonetheless, TCM was challenged by western medicine because most assessed therapies and effective ingredient remain uncertain. Furthermore, most of verification trials were published in Chinese, inaccessible to western researchers, and not served in systematic reviews (Tang et al., 2008).

Dysfunctional uterine bleeding (DUB), which is characterizing as excessively heavy, prolonged and frequent bleeding of uterine origin, is a common disease to the Emergency and Gynecology Department (Deligeoroglou et al., 2013). The primary cause of DUB is the dysfunction of the HPO (Hypothalamuspituitary-ovarian) axis (Coll Capdevila, 1997), which is accounted for the expression of female hormones, including follicle-stimulating hormone $(\mathrm{FSH})$, luteinizing hormone $(\mathrm{LH})$, progesterone $(\mathrm{P})$, and estrogen $(\mathrm{E})$ (Afrin et al., 2019; Kho and Mathur, 2015). The sudden decrease of E level in the DUB patients induces apoptosis of epithelial cells of endometrial glands, resulting in irregular shedding of endometrium (Deligeoroglou et al., 2013). TCM treatments studies have shown promising results for delayed menstrual cycles to cure the DUB (Penn, 2018). Furthermore, most of western medicine treatments are given priority 
to oral sex hormone drugs and injections, such as nonsteroidal anti-inflammatory drugs, tranexamic acid, prostaglandins, estrogen (Benetti-Pinto et al., 2017; Borzutzky and Jaffray, 2019). However, most drugs have certain side effects, which are accompanied by nausea, vomiting, and headaches (Bianchi Porro and Parente, 1989; FitzGerald, 2002; Grosser et al., 2017). Hence, it is essential to promote the development of new effective drugs for the cure of DUB patients.

Several drugs and extractions have been demonstrated to play a key role in strengthening coagulation, reducing inflammation and lowering uterine bleeding in the DUB patients. Steroidal glycosides, extracted from the berries of Solanum nigrum $L$., have been confirmed to enhance the ability of anti-inflammatory in injured cells (Xiang et al., 2018). Besides, Gongxuening capsule, a certified Chinese patent medicines with curative effects on DUB, can strengthen platelet aggregation, hemostasis and coagulation function, thereby increasing female estrogen and progesterone levels and regulating HPO axis function (Liu et al., 2009). Here, we identified that an unexploited Chinese recipe, Xuebeng recipe (XBR), is able to possess positive effects on the treatment of DUB. XBR not only harbors the function of hemostasis and coagulation, but also regulates proliferative endometrium at the hormone and gene expression levels.

\section{Materials And Methods}

Drugs. XBR drug, a blended traditional Chinese medicine, consisting of Saururus chinensis, Celosia cristata and Spatholobus suberectus, was performed in the study following the stipulation of Chinese Pharmacopoeia 2010 (Zhou et al., 2012). The quality control of the drug was determined by the thin layer chromatography and high-performance liquid chromatography, and the drug dose conversion and preparation were displayed in detail in the supplementary document. XBR and positive control drugs Gongxuening (Yunnan Baiyao Group Co., Ltd.) were administrated daily by intragastric gavage in the Sprague-Dawley (SD) rats.

Animals and experimental diets. Female SD rats purchased from the Hunan SJA Laboratory Animal Co., Ltd (Changsha, China) were used in all of the experiments. The SD rats were kept in specific pathogenfree conditions in the animal experiment center of Hunan Normal University. The diets of the rats were provided with Grade B rat breeding stocks daily, and the rats had ad libitum access to food and water at all times during the study.

Animal experiment I. Healthy SD female rats, weighing $130 \pm 20 \mathrm{~g}$, were habitually reared for one week and randomly divided into the following two groups: blank control group (CK, intragastrically administered equal volume of normal saline), and secretion 20-dose administration group (XBR 20x). Four SD female rats per cage were administered intragastrically at a fixed time every day. The animal response was closely observed within $2 \mathrm{~h}$ after the intragastric administration. Feed $25 \mathrm{~g} / \mathrm{d}$ each, drink naturally, and control light for $12 \mathrm{~h}$ every day. For 7 consecutive days, pay attention to and record the poisoning and death. Furthermore, calculate the effects of excessive XBR drugs on visceral coefficients and blood biochemical parameters in the SD rats. The rats were then sacrificed under light ether anesthesia. 
Animal experiment II. Healthy SD female rats, weighing $200 \pm 20 \mathrm{~g}$, were adaptively reared for one week and randomly divided into the following three groups: blank control group (CK, gavage of equal volume of normal saline), Gongxuening positive control group (GXN), XBR 5-dose group (XBR). According to the drug ratio relationship (see Additional file 1: Table S1), the saline was uniformly used to prepare the suspension for gavage. Four SD female rats per cage were administered intragastrically at a fixed time every day for $30 \mathrm{~d}$. The animal response was closely observed within $2 \mathrm{~h}$ after intragastric administration, and death were noted and recorded.

Thirty minutes after the end of gavage on the 30 th $\mathrm{d}$, the tail was cut off $0.5 \mathrm{~cm}$ from the tip of the rat, and the time was counted from the blood flowed out spontaneously to the tail tip stop bleeding. Meanwhile, use a clean pin every $15 \mathrm{~s}$ to pick up the bottom of the blood drop until the fibrin filaments showed up to record the clotting time. Furthermore, each rat's right foot was injected with $0.1 \mathrm{~mL}$ of a $10 \%$ egg white saline (EWS). The foot volume of the rats was recorded at $10 \mathrm{~min}, 20 \mathrm{~min}$, and $30 \mathrm{~min}$ after the injection, and the foot swelling volume data was obtained to calculate the detumescent degree of the rats. The rats were then sacrificed under light ether anesthesia.

Animal experiment III. Healthy SD female rats, weighing $200 \pm 20 \mathrm{~g}$, were reared adaptively for one week and randomly divided into blank control group $(C K, n=8)$ and model group $(n=24)$. The rats in the model group were intramuscularly injected with estradiol benzoate $1 \mathrm{mg} / \mathrm{kg}$ every other day to yield endometrial hyperplasia (EH) model rats. After 60 d injections, the EH model rats were randomly divided into 3 groups: $\mathrm{EH}$ model group (EH, gavage of equal volume of normal saline), GXN group and XBR group. The two different drugs (Dosage is the same as experiment II) were uniformly prepared with saline for suspension, and the CK group was intramuscularly injected with $0.9 \%$ sodium chloride injection. On the $0 \mathrm{~d}$, the $3 \mathrm{~d}$ and the $7 \mathrm{~d}$ of administration, four rats were randomly selected from each group to test the serous biochemical indexes and enzyme activity. The histological alterations in the endometrium were detected by scanning electron microscopy (SEM).

Moreover, 12 SD rats received a daily intravenous injection of $20 \mathrm{~g} / \mathrm{L}$ of a high-molecular-weight gluconic anhydride solution to yield blood stasis (BS) model rats. After continuous injection for $3 \mathrm{~d}$, they were randomly divided into 3 groups: BS model group (BS), GXN group and XBR group. The gavage procedures were the same as the above experiment for $7 \mathrm{~d}$. The rats were sacrificed after the continuous administration, and the blood was collected in a $3 \mathrm{~mL}$ heparin-containing centrifuge tube to further identify the blood viscosity.

Animal experiment IV. The preparation, grouping and drug treatment of EH rats were the same as Animal Experiment III. As previously mentioned (Coll Capdevila, 1997), four crucial hormones regulated by the $\mathrm{H}$ P-O axis are related to endometrial hyperplasia. Hence, the expression activity of progesterone (P), estradiol (E2), follicle-stimulating hormone (FSH) and luteotropic hormone (LH) in the blood of SD rats was detected by the enzyme-linked immunoassay (ELISA) kit following the manufacturing requirements.

In addition, the ovaries and uterus were taken immediately after dissection of rats, and the mRNA expression levels of matrix metalloproteinase-1 (MMP-1) and tissue inhibitor of matrix metalloproteinase- 
1 (TIMP-1) were detected by RT-qPCR technology. The primer design was listed the supplementary document (Additional file 2: Table S2).

\section{Results}

Animal Experiment I: High-dose XBR formulation exerted no significant toxic effect on the SD rats. The rats were closely observed after daily administration, and the results represented that no abnormal reactions and body weight alterations (Fig. 1A) were found. The organ coefficient of animals is closely related to the growth and development of organs (Zhang et al., 2014). While in this XBR limit test, the value of the spleen, kidney, and genital did not alter significantly $(p>0.05)$, but the index of liver increased significantly $(p<0.05)$ (Fig. 1B), indicating that high-concentration medication may induce certain load to the live. The levels of alanine aminotransferase (ALT) and aspartate aminotransferase (AST) in the serum of XBR 20x rats were increased dramatically compared with the CK group (Fig. 1C), but the ratio of ALT/AST displayed no significant difference $(p>0.05)$.

Animal Experiment II: XBR drug conserved coagulation and toe edema time in the SD rats. Compared with the CK and GXN, only XBR harbored an accelerated blood coagulation effect on tail-broken rats (Fig. 2A). Similar alterations in the hemostatic time were also observed in the SD rats, but did not display a significant difference (Fig. 2B). Moreover, after injection with EWS, the relative toe edema in XBR rats reduced dramatically at 20 and 30 min comparing with $10 \mathrm{~min}$, and the drugs represented distinctively comparing with other experiment group (Fig. 2C).

Notably, The XBR drugs in the experiment showed no alteration in the liver coefficient comparing with CK group, and the expression level of total protein (TP) and AST altered significantly while other detected biomarkers remained unchanged (Additional file 3: Fig S1). Moreover, the hematic phase results indicated that the content of erythrocyte, hemoglobin and hematokrit showed significant down-regulated differences between CK and XBR rats, indicating the XBR drugs may reduce the possibility of blood stasis (Additional file 4: Table S3).

\section{Animal Experiment III: XBR drug restored the proliferating endometrium in SD rats to normal and induced erythrocyte aggregation. After the 60th $d$ of modelling (0th $d$ of administration), the anatomy of rats was randomly selected from the CK (Fig. 3A) and the model group (Fig. 3B). After 3 and $7 \mathrm{~d}$ of administration, most of the pinopodes (a morphological biomarker of endometrial epithelial cells) in the EH (Fig. 3C, 3F) and GXN (Fig. 3D, 3G) were atrophic, hollow and sparsely arranged, while the pinopodes in the XBR cells gradually recovered to rounded (Fig. 3E, 3H).}

On the $3 \mathrm{~d}$ of administration, the thickness of the endometrium in the XBR rats was significantly reduced, and it showed extremely different from the rats of other experimental groups on the $7 \mathrm{~d}$ (Fig. 4A). Interestingly, the endometrial thickness of the XBR gradually recovered to the state of $\mathrm{CK}$ rats. The biochemical parameters in the 3 and $7 \mathrm{~d}$ of the SD rats showed the alterations in the albumin (ALB), total cholesterol (TC) and ALT/AST (Fig. 4C D), indicating that XBR may increase the protein and cholesterol levels in serum of endometrial hyperplasia rats. The blood viscosity results showed that the plasma 
concentration (PC) of the BS group was significantly higher than CK rats, while the whole blood highshearing relative index (WBHRI) and whole blood low-shearing relative index (WBLRI) exhibited no significant difference, and the erythrocyte aggregation index (EAI) was higher $(p<0.05)$ in the serum of XBR rats (Fig. 4B).

Notably, pathological section of rat kidney showed that the number of glomeruli and tubules in the XBR group was higher than that of other rats (Additional file 5: Fig S2). Liver pathological section results showed that gap junctions between the hepatocytes in the XBR group increased after $3 \mathrm{~d}$ of treatment. Nonetheless, the cell junction gap and the number of eosinophils in all experimental rats did not alter significantly on the $7 \mathrm{~d}$, indicating that the XBR exerted no significant side effects in the rat liver.

\section{Animal experiment IV. XBR drugs altered the hormone expression ( $F S H, L H, E_{2}$ and $P$ ) and gene} expression (MMP-1 and TIMP-1) in the endometrial hyperplasia rats. The results showed that the FSH content in the XBR rats increased gradually with gavage time, and was significantly higher than that in CK, EH and GXN groups at day 7 (Fig. 5A). Interestingly, both LH concentrations in GXN and XBR rats returned to normal after a short-term rise (Fig. 5B). Coupled with these are the downtrend of $E_{2}$ (Fig. 5C) and uptrend of $\mathrm{P}$ (Fig. 5D). In addition, XBR significantly regulated the expression of MMP-1 gene, and its significance is higher than that of other experimental groups (Fig. 6A). Similarly, the ascending expression of TIMP-1 gene in the XBR rats was observed, and the expression level is dramatically higher than other groups (Fig. 6B).

\section{Discussion}

Taking into account the characteristics of TCM with low toxicity and difficulty in measuring lethal dose (Liu et al., 2015), thus the safety concerns such as treatment duration, long-term toxicity and dosedependent toxicity need to be carefully evaluated in the use of TCM. Indeed, several herbs have been demonstrated to cause severe intrinsic renal, hepatic, neurologic and carcinogenic toxicities (Haller et al., 2002; Nyirimigabo et al., 2015; Shaw, 2010; Teschke et al., 2014). Though some studies indicate that many species of Aristolochia in TCM were employed to treat symptoms such as acute arthritis and edema, but aristolochic acid extracted from the herb was found to contribute to renal impairment (Chen et al., 2013; Vanherweghem, 1998). Hence, before the drug is officially used, limit experiments need to be performed to detect the toxicity of XBR on rats. The results from the Animal Experiment I elucidate that 20-dose XBR exerted no toxic effect on physiological parameters such as body weight in the rats. Nonetheless, the organ index of rat liver changed significantly, suggesting that high doses of the drug might have some effect on the rat liver, while the index returned to normal at 5-dose XBR (Fig. 2A). Furthermore, both ALT and AST are important biomarkers of liver function testing (Liu et al., 2018), but the significance of the disease course and prognosis can only be determined when the ALT / AST ratio is changed (Khattab et al., 2015). Therefore, notwithstanding the contents of AST and AST in the blood of rats have altered significantly in the limit experiment, the value is still in the safe range. Interestingly, liver histologic sections showed increased gap junctions between hepatocytes in the XBR rats, indicating that the drug can elevate liver metabolic activity in the model rats. In addition, XBR exerted no significant 
impact on other organ indexes (kidney, spleen, and genitals), it is therefore can basically be determined that XBR has no obvious toxic effect on SD rats.

DUB can cause a range of bleeding symptoms, particularly a frequent and/or uncontrolled bleeding of uterine, it is therefore necessary to identify the hemostatic, procoagulant and antiphlogistic effects of the drug. Total protein (TP) in the serum is composed of two main components, albumin (ALB) and globulin (GLO) (Alkan et al., 2015; Guo et al., 2018). ALB is a natural part of human blood, which has the function of protecting blood cells, platelets, and regulating coagulation, while globulin also has anti-inflammatory and antibacterial effects (Eljaiek et al., 2017). After the XBR administration, it was found that the TP content (Fig. 2B) and ALB content (Fig. 4D) in the rat serum were significantly increased, indicating that the drug can induce the increase of antibacterial substances in the serum of rats, thereby exerting coagulation and anti-inflammatory effects. The symptoms of hyperviscosity are increased hematocrit and red blood cell count, while the value of erythrocyte, hemoglobin, and hematocrit decreased remarkably in the table 1 , indicating that XBR regulated blood substances and reduced blood viscosity in the rats, thus reducing the occurrence of blood stasis.

Normal uterine bleeding is caused by periodic changes in the endometrium, which is regulated via the HPO axis. The morphological variations of the functional layer of the endometrium can be divided into three phases, namely the proliferative phase, secretory phase, and menstrual phase (Tanos et al., 2020). During the menstrual period, the levels of $E$ and $P$ dropped, which subsequently causes the endometrial blood flow to decrease, resulting in endometrial necrosis due to ischemia. The area of the damaged and ischemic necrotic tissue gradually expanded, the permeability of the blood vessel wall boosted, and finally the tissue was exfoliated (Jarrell, 2018; Lichten, 2018). Anovulatory DUB due to the effects of prolonged $E$ and no progestin' s counteracting effect, the endometrium continues to proliferate and even proliferate to varying degrees, eventually leading to breakthrough-estrogen-bleeding (Jones and Sung, 2020; Urbanska et al., 2019). What's worse, it is difficult to identify the location, depth, scope and timing of the degradation and fall. Interestingly, our findings demonstrated that the pinopodes of EH rats can gradually return to functional morphology after XBR administration, which proves that the drug has an inhibitory effect on endometrial hyperplasia and is much better than the contrastive drug GXN. Hence, the authors suggest that XBR can not only enhance the coagulation and anti-inflammatory ability of rats, reduce the occurrence of blood stasis, but also control the endometrial hyperplasia thus lessen the bleeding caused by the endometrium shedding.

Gonadotropin-releasing hormone secreted by the hypothalamus can induce the synthesis of FSH and LH, thereby promoting follicular development and increasing E secretion (Das and Kumar, 2018; Filatov et al., 2017; Smitz et al., 2016). LH increases $P$ secretion and leads to ovulation. With the increase of $E$ and $P$ levels, it will produce negative feedback inhibition on the hypothalamus and pituitary gland, thus reducing the levels of FSH and LH, leading to luteal degradation (Di Renzo et al., 2016; Utian, 1989; Ye et al., 2018). Menstruation occurs when the endometrium loses the support of these two hormones and exfoliates and bleeds. The imbalance of FSH and LH secretion will affect the production of $E_{2}$ and $\mathrm{P}$, and then affect the two-way feedback regulation of sex hormones, which causes female functional uterine bleeding 
(Abdollahi et al., 2018; Chuah et al., 2017). Estrogen can regulate uterine development and stimulate endometrium hyperplasia and thickening, while $\mathrm{P}$ can stimulate endometrium proliferation under the stimulation of $\mathrm{E}$. If the expression level of both increases simultaneously, it will cause endometrium thickening and may cause functional uterine bleeding. Hence, we speculate whether the inhibited hyperplasia of the endometrium is due to XBR regulating the related hormone expression levels in the rats. Test results echoed our opinion: After administration of XBR, the levels of FSH in female rats elevated, LH increased first and then decreased, E2 declined, and P gradually facilitated. The result reflects the negative feedback mechanism of HPO, and the content of E and P did not boost dramatically together, thereby lowing the emergence of endometrial hyperplasia.

MMPs are a group of proteases that degrade the extracellular matrix (ECM), and TIMPs can specifically bind to activated MMPs to inhibit its activity and prevent further degradation of ECM (Arpino et al., 2015; Cui et al., 2017). Studies have shown that the MMPs and TIMPs changed distinctively in ectopic endometrial patients (Uzan et al., 2004). The imbalance between MMPs and TIMPs promote the degradation of ECM, and increase the invasiveness of ectopic endometrium (Collette et al., 2004). Endometrial hyperplasia results from prolonged stimulation of the endometrium with unopposed estrogen, while many studies have shown that MMPs have significant differential expression in uterine fibroids, including high-expression of MMP-1, MMP-2, MMP-9 and low-expression of MT1-MMP, TIMP-1,-2, and-3 (lurlaro et al., 1999; Knox et al., 1996; Maatta et al., 2000; McDonnell et al., 1991). MMPs promote sustained cellular growth by activating growth factors, inducing growth factor binding proteins, and releasing mitogenic molecules from the ECM (Pilka et al., 2004). In addition, MMPs and TIMPs are not only enzymes that regulate ECM, but also play an important role in organogenesis, angiogenesis, and wound repair (Hu et al., 2019; Liu et al., 2016; Smane-Filipova et al., 2016; Xing et al., 2017). In the present study, it is reported that there was a significant difference in the expression of MMP-1 and TIMP-1 in differently treated rats after $7 \mathrm{~d}$ gavage. Though the expression tendency of MMP-1 in XBR rats was downtrend, the relative expression levels were significantly higher than other treatment groups, indicating that XBR can induce the expression of MMP-1 gene thereby promoting the degradation of ECM and reduce the endometrial hyperplasia.

\section{Conclusion}

In conclusion, this study provided evidence that alterations to the hormones and mRNA induced by gavage of XBR could affect the endometrial hyperplasia of the rats. Under this mechanism, the endometrium will not cause frequent bleeding due to cell necrosis and shedding. In addition, XBR drug can reduce blood stasis, enhance coagulation and anti-inflammatory effects, thus providing a protective mechanism in the event of normal bleeding. Hence, understanding the molecular mechanisms of drugdisease interactions may construct prospective strategies for the treatment of DUB patients and play a great role in the promotion of Chinese medicine.

\section{Declarations}




\section{Associated content}

\section{Supporting Information}

Detailed methods for the process of medication, quality determination and animal trials were listed in the document. Effects of XBR medication on organ coefficients and blood biochemical parameters (Fig S1); Kidney and liver histologic sections of endometrial hyperplasia rats (Fig S2); Three Chinese medicine ingredients were corresponded to the control medicine (Fig. S3); Biomarker (sauchinone) was observed in both S. chinensis and control drugs (Fig S4); Biomarker (formononetin) was observed in both $S$. suberectus and control drugs (Fig S5); Drug conversion relationship between human and rat (Table S1); Primer design for qPCR (Table S2); Effects of XBR on the hematic phase in rats (Table S3).

\section{Conflict of interest}

The authors declare that they have no conflict of interest.

\section{Acknowledgement}

This work was supported by the National Natural Science Foundation of China (Nos. 31472017, 31272339), Project of Science and Technology Department of Hunan Province (No.2014FJ2003), Tropical Disease Control and Research, Ministry of Education in China (2018kfkt03), Scientific Research Project of Hunan Provincial Education Department (16C0778), and Key Project of Education Department of Hunan Province (18A024).

\section{References}

Abdollahi, N. G., et al., 2018. The effects of fennel on menstrual bleeding: A systematic review and metaanalysis. J Complement Integr Med. 15.

Afrin, L. B., et al., 2019. Successful mast-cell-targeted treatment of chronic dyspareunia, vaginitis, and dysfunctional uterine bleeding. J Obstet Gynaecol. 39, 664-669.

Alkan, A., et al., 2015. Albumin to globulin ratio, a predictor or a misleader? Ann Oncol. 26, 443-4.

Arpino, V., et al., 2015. The role of TIMPs in regulation of extracellular matrix proteolysis. Matrix Biol. 44$46,247-54$.

Bai, Z. H., et al., 2015. Eye acupuncture treatment for stroke: a systematic review and meta-analysis. Evid Based Complement Alternat Med. 2015, 871327.

Benetti-Pinto, C. L., et al., 2017. Abnormal uterine bleeding. Rev Bras Ginecol Obstet. 39, 358-368.

Bianchi Porro, G., Parente, F., 1989. Side effects of anti-ulcer prostaglandins: an overview of the worldwide clinical experience. Scand J Gastroenterol Suppl. 164, 224-9; discussion 229-31. 
Borzutzky, C., Jaffray, J., 2019. Diagnosis and management of heavy menstrual bleeding and bleeding disorders in adolescents. JAMA Pediatr.

Chen, C. H., et al., 2013. Aristolochic acid-induced upper tract urothelial carcinoma in Taiwan: clinical characteristics and outcomes. Int J Cancer. 133, 14-20.

Chuah, I., et al., 2017. Menstrual management in developmentally delayed adolescent females. Aust N Z J Obstet Gynaecol. 57, 346-350.

Coll Capdevila, C., 1997. Dysfunctional uterine bleeding and dysmenorrhea. Eur J Contracept Reprod Health Care. 2, 229-37.

Collette, T., et al., 2004. Evidence for an increased release of proteolytic activity by the eutopic endometrial tissue in women with endometriosis and for involvement of matrix metalloproteinase-9. Hum Reprod. 19, 1257-64.

Cui, N., et al., 2017. Biochemical and biological attributes of matrix metalloproteinases. Prog Mol Biol Transl Sci. 147, 1-73.

Das, N., Kumar, T. R., 2018. Molecular regulation of follicle-stimulating hormone synthesis, secretion and action. J Mol Endocrinol. 60, R131-R155.

Deligeoroglou, E., et al., 2013. Abnormal uterine bleeding and dysfunctional uterine bleeding in pediatric and adolescent gynecology. Gynecol Endocrinol. 29, 74-8.

Di Renzo, G. C., et al., 2016. Progesterone in normal and pathological pregnancy. Horm Mol Biol Clin Investig. 27, 35-48.

Eisenhardt, S., Fleckenstein, J., 2016. Traditional Chinese medicine valuably augments therapeutic options in the treatment of climacteric syndrome. Arch Gynecol Obstet. 294, 193-200.

Eljaiek, R., et al., 2017. Albumin administration for fluid resuscitation in burn patients: A systematic review and meta-analysis. Burns. 43, 17-24.

Filatov, M., et al., 2017. Influence of gonadotropins on ovarian follicle growth and development in vivo and in vitro. Zygote. 25, 235-243.

FitzGerald, G. A., 2002. Cardiovascular pharmacology of nonselective nonsteroidal anti-inflammatory drugs and coxibs: clinical considerations. Am J Cardiol. 89, 26D-32D.

Gan, T., et al., 2010. Traditional Chinese Medicine herbs for stopping bleeding from haemorrhoids. Cochrane Database Syst Rev. CD006791.

Grosser, T., et al., 2017. The Cardiovascular pharmacology of nonsteroidal anti-inflammatory drugs. Trends Pharmacol Sci. 38, 733-748. 
Guo, H. W., et al., 2018. Prognostic value of pretreatment albumin/globulin ratio in digestive system cancers: A meta-analysis. PLoS One. 13, e0189839.

Haller, C. A., et al., 2002. Making a diagnosis of herbal-related toxic hepatitis. West J Med. 176, 39-44.

He, K., 2015. Traditional Chinese and Thai medicine in a comparative perspective. Complement Ther Med. 23, 821-6.

$\mathrm{Hu}, \mathrm{X}$., et al., 2019. Disequilibrium in MMPs and the tissue inhibitor of metalloproteinases in different segments of the varicose great saphenous vein wall. Int Angiol. 38, 185-193.

lurlaro, M., et al., 1999. Angiogenesis extent and expression of matrix metalloproteinase-2 and -9 correlate with upgrading and myometrial invasion in endometrial carcinoma. Eur J Clin Invest. 29, 793-801.

Jarrell, J., 2018. The significance and evolution of menstruation. Best Pract Res Clin Obstet Gynaecol. 50, 18-26.

Jones, K., Sung, S., Anovulatory Bleeding. StatPearls, Treasure Island (FL), 2020.

Khattab, H., et al., 2015. Relation of ALT and AST levels to the histopathological changes in liver biopsies of patients with chronic hepatitis C genotype 4. Arab J Gastroenterol. 16, 50-3.

Kho, C. L., Mathur, M., 2015. Uterine artery embolisation for acute dysfunctional uterine bleeding with failed medical therapy: a novel approach to management. BMJ Case Rep. 2015.

Knox, J. D., et al., 1996. Matrilysin expression in human prostate carcinoma. Mol Carcinog. 15, 57-63.

$\mathrm{Li}$, J., et al., 2018. Combination of artemisinin-based natural compounds from Artemisia annua L. for the treatment of malaria: Pharmacodynamic and pharmacokinetic studies. Phytother Res. 32, 1415-1420.

Lichten, E. M., 2018. Menstrual migraine and treatment options: Review. Headache. 58, 145-146.

Liu, Q., et al., 2009. A simple and rapid method for the determination of pennogenin diglycoside in rat plasma by HPLC-MS: application to the pharmacokinetics of the extract in Gongxuening capsules. J Chromatogr Sci. 47, 728-32.

Liu, S. H., et al., 2015. Safety surveillance of traditional Chinese medicine: current and future. Drug Saf. $38,117-28$.

Liu, Y., et al., 2016. Association of MMPs and timps with the occurrence of atrial fibrillation: a systematic review and meta-analysis. Can J Cardiol. 32, 803-13.

Liu, Y., et al., 2018. AST to ALT ratio and arterial stiffness in non-fatty liver Japanese population:a secondary analysis based on a cross-sectional study. Lipids Health Dis. 17, 275. 
Maatta, M., et al., 2000. Localization of MT1-MMP, TIMP-1, TIMP-2, and TIMP-3 messenger RNA in normal, hyperplastic, and neoplastic endometrium. Enhanced expression by endometrial adenocarcinomas is associated with low differentiation. Am J Clin Pathol. 114, 402-11.

McDonnell, S., et al., 1991. Expression and localization of the matrix metalloproteinase pump-1 (MMP-7) in human gastric and colon carcinomas. Mol Carcinog. 4, 527-33.

Nyirimigabo, E., et al., 2015. A review on phytochemistry, pharmacology and toxicology studies of Aconitum. J Pharm Pharmacol. 67, 1-19.

Penn, Y. Y., 2018. Acupuncture treatment for dysfunctional uterine bleeding in an adolescent. BMJ Case Rep. 2018.

Pilka, R., et al., 2004. Matrix metalloproteinase-26 (matrilysin-2) expression is high in endometrial hyperplasia and decreases with loss of histological differentiation in endometrial cancer. Gynecol Oncol. $94,661-70$.

Shaw, D., 2010. Toxicological risks of Chinese herbs. Planta Med. 76, 2012-8.

Smane-Filipova, L., et al., 2016. MMPs and TIMPs expression in facial tissue of children with cleft lip and palate. Biomed Pap Med Fac Univ Palacky Olomouc Czech Repub. 160, 538-542.

Smitz, J., et al., 2016. Follicle-stimulating hormone: a review of form and function in the treatment of infertility. Reprod Sci. 23, 706-16.

Tang, J. L., et al., 2008. Traditional Chinese medicine. Lancet. 372, 1938-40.

Tanos, V., et al., 2020. Junctional zone endometrium morphological characteristics and functionality: review of the literature. Gynecol Obstet Invest. 1-11.

Teschke, R., et al., 2014. Review article: Herbal hepatotoxicity--an update on traditional Chinese medicine preparations. Aliment Pharmacol Ther. 40, 32-50.

Tu, Y., 2016. Artemisinin-a gift from traditional Chinese medicine to the world (Nobel Lecture). Angew Chem Int Ed Engl. 55, 10210-26.

Urbanska, E., et al., 2019. Is polycystic ovarian syndrome and insulin resistance associated with abnormal uterine bleeding in adolescents? Ginekol Pol. 90, 262-269.

Utian, W. H., 1989. Biosynthesis and physiologic effects of estrogen and pathophysiologic effects of estrogen deficiency: a review. Am J Obstet Gynecol. 161, 1828-31.

Uzan, C., et al., 2004. Eutopic endometrium and peritoneal, ovarian and bowel endometriotic tissues express a different profile of matrix metalloproteinases-2, -3 and -11 , and of tissue inhibitor metalloproteinases-1 and -2. Virchows Arch. 445, 603-9. 
Vanherweghem, L. J., 1998. Misuse of herbal remedies: the case of an outbreak of terminal renal failure in Belgium (Chinese herbs nephropathy). J Altern Complement Med. 4, 9-13.

Xiang, L., et al., 2018. Anti-inflammatory steroidal glycosides from the berries of Solanum nigrum L. (European black nightshade). Phytochemistry. 148, 87-96.

Xiao, E., Luo, L., 2018. Alternative therapies for diabetes: a comparison of western and traditional Chinese medicine (TCM) approaches. Curr Diabetes Rev. 14, 487-496.

Xing, Y., et al., 2017. MMPs/TIMPs imbalances in the peripheral blood and cerebrospinal fluid are associated with the pathogenesis of HIV-1-associated neurocognitive disorders. Brain Behav Immun. 65, 161-172.

Yang, L., et al., 2018. Warm-needle moxibustion for spasticity after stroke: A systematic review of randomized controlled trials. Int J Nurs Stud. 82, 129-138.

Ye, H., et al., 2018. Intimate estrogen receptor-alpha/ligand relationships signal biological activity. Toxicology. 408, 80-87.

Zhang, R., et al., 2014. Mouse organ coefficient and abnormal sperm rate analysis with exposure to tap water and source water in Nanjing reach of Yangtze River. Ecotoxicology. 23, 641-6.

Zhou, Y., et al., 2012. High performance liquid chromatographic separation of thirteen drugs collected in Chinese Pharmacopoeia 2010(Ch.P2010) on cellulose ramification chiral stationary phase. J Pharm Anal. 2, 48-55.

\section{Table 1}

Table 1 is not available in this version.

\section{Figures}



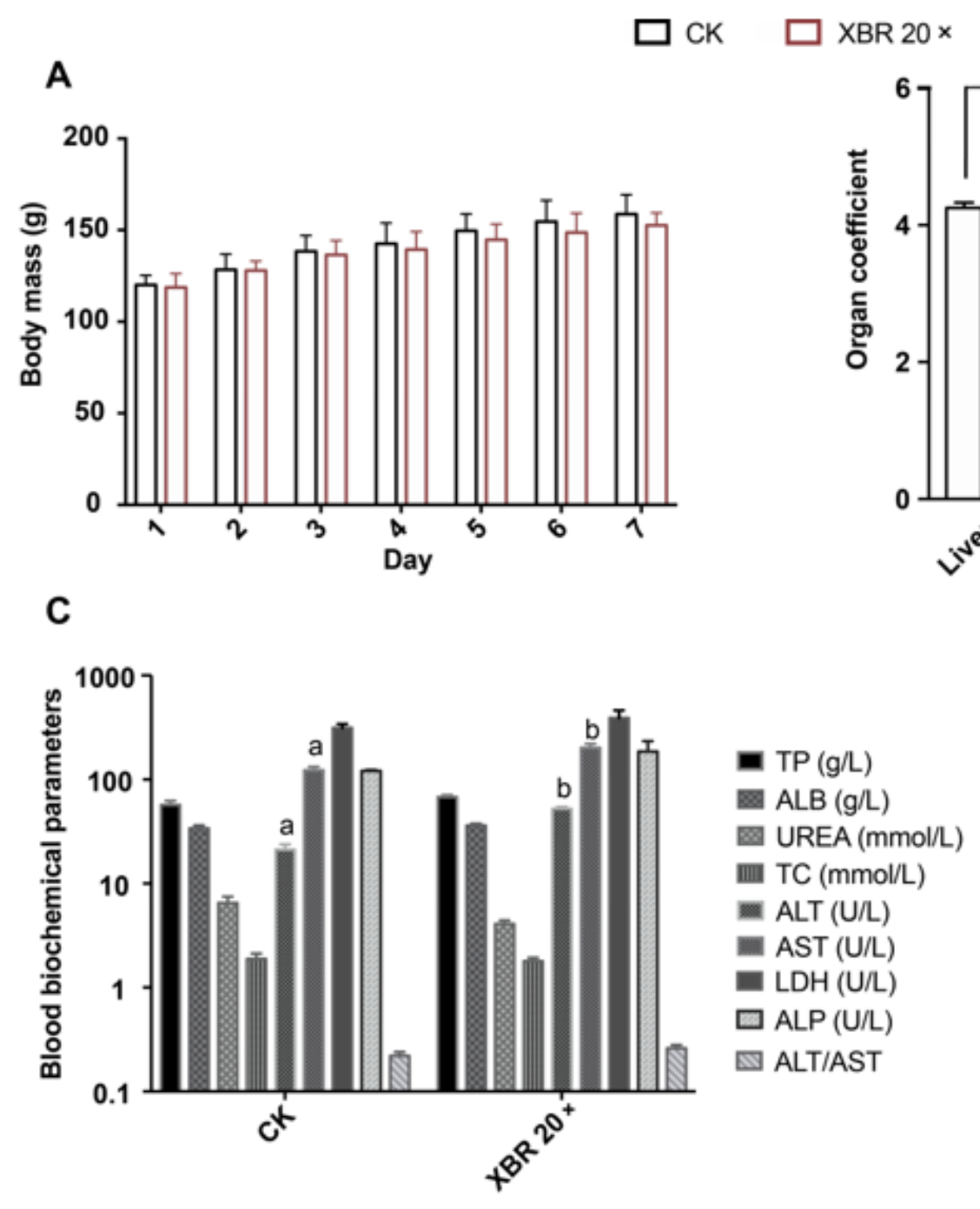

Figure 1

The effects of high-dose XBR drug on the body mass, organ index and blood biochemical parameters of SD rats. (A) Histogram of body mass alterations in high-dose XBR treated rats. (B). Effects of high-dose medication on organ coefficients in rats. Organ coefficient expressed by dividing the organ weight by body mass. (C) The alterations of blood biochemical parameters under the gavage of high-dose XBR. Values are means \pm SEM. ab Different superscripts or * asterisk indicate the significant difference $(\mathrm{p}<$ 0.05 , t-Student test, $n=4$ ). Abbreviations: TP, total protein; ALB, albumin; TC, total cholesterol; ALT, alanine aminotransferase; AST, aspartate aminotransferase; LDH, lactic dehydrogenase; ALP, alkaline phosphatase. Similarly hereinafter the same below. 

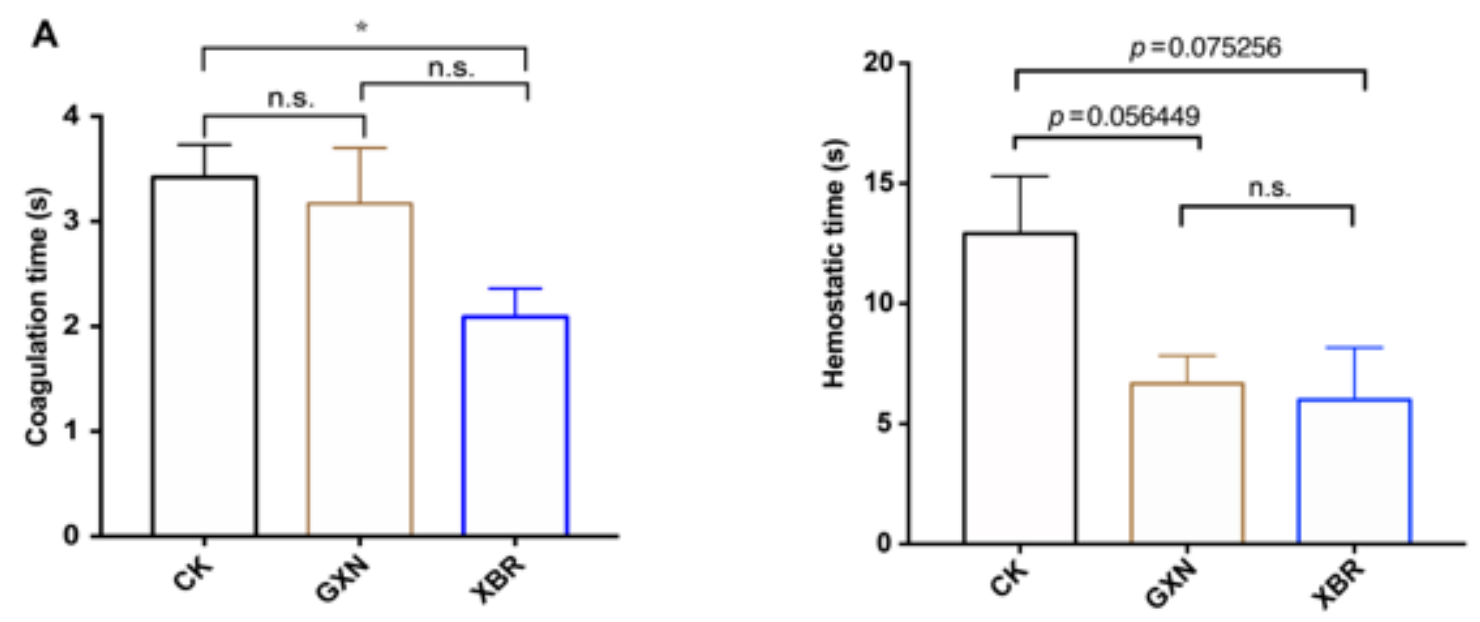

B

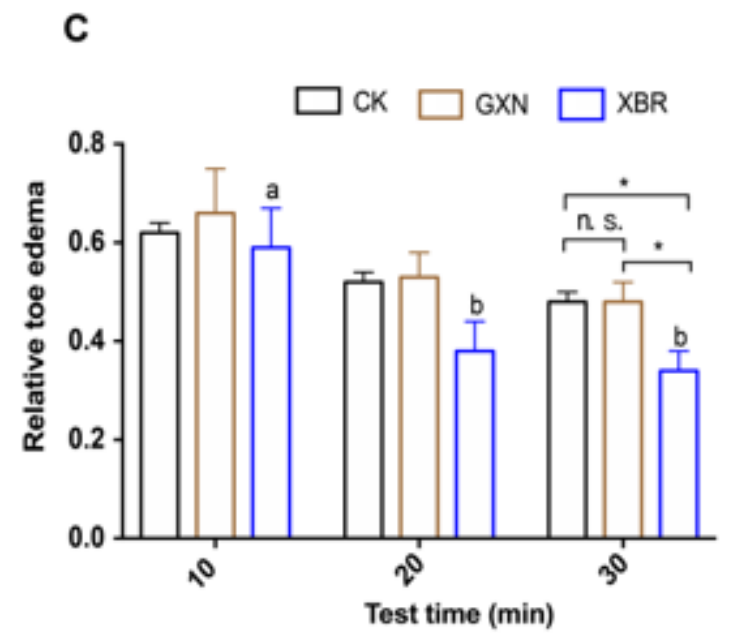

\section{Figure 2}

The effects of XBR drug on the hemostasis, coagulation and anti-inflammation of SD rats. (A) Effects of different medical treatments on coagulation time. (B) Effects of different medical treatments on hemostatic time. (C) The changes of paw detumescence. Relative toe edema expressed by (toe volume after swelling - toe volume before swelling) / toe volume before swelling. Values are means \pm SEM. ab Different superscripts or * asterisk indicate the significant difference $(p<0.05$, t-Student test, $n=4)$. 

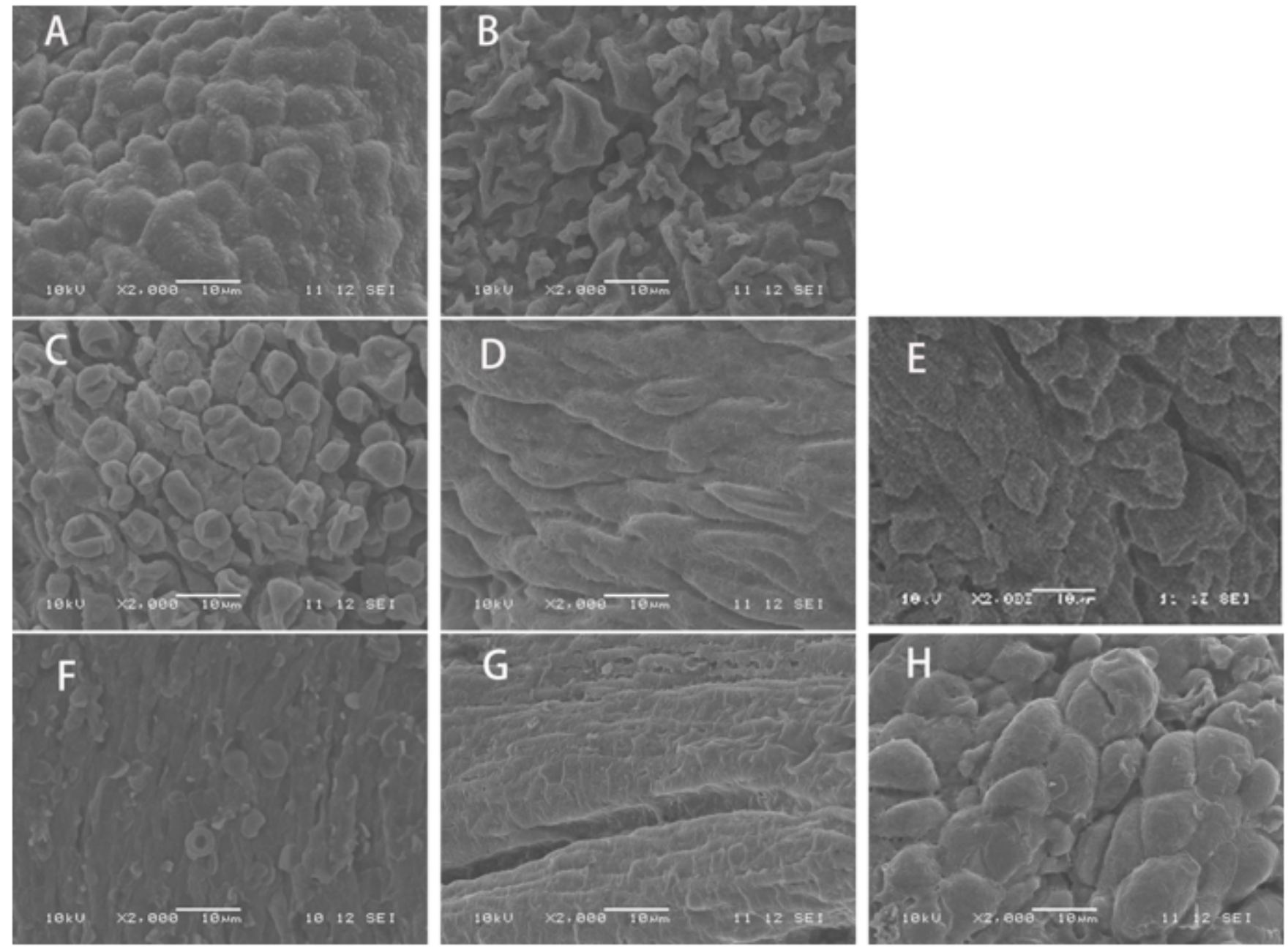

\section{Figure 3}

Scanning electron microscope (SEM) images of rat endometrium. (A) SEM image of CK rat endometrium. (B) SEM image of estradiol benzoate treated rat endometrium. (C, D, E) SEM images of rat endometrium after $3 \mathrm{~d}$ of gavage (C EH, D GXN, E XBR). $(F, G, H)$ SEM images of rat endometrium after $7 \mathrm{~d}$ of gavage (F EH, G GXN, H XBR). 


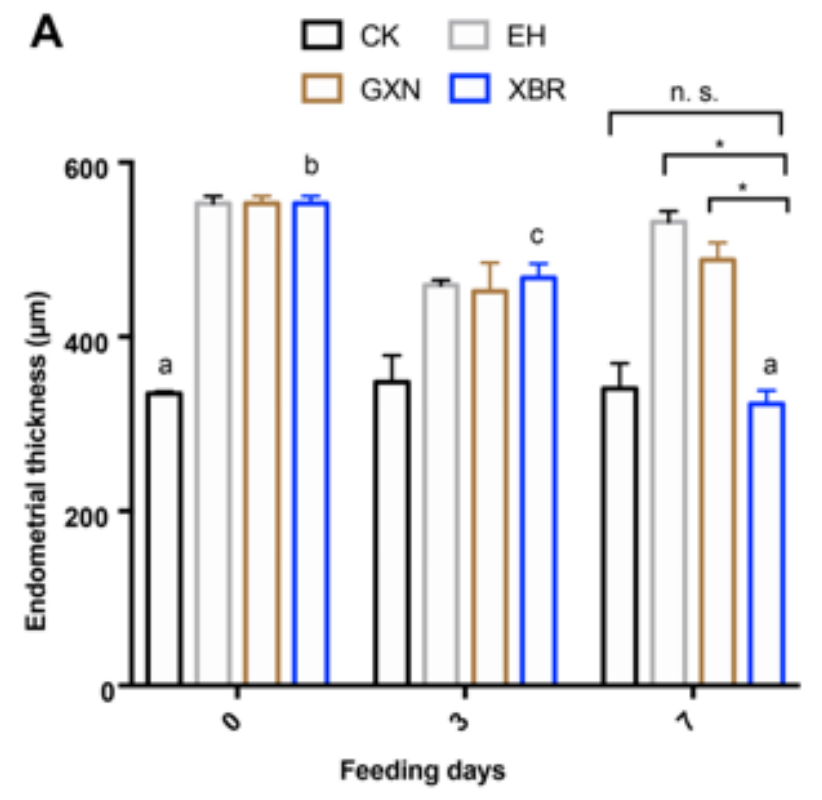

W CK $\mathrm{EH}$

B
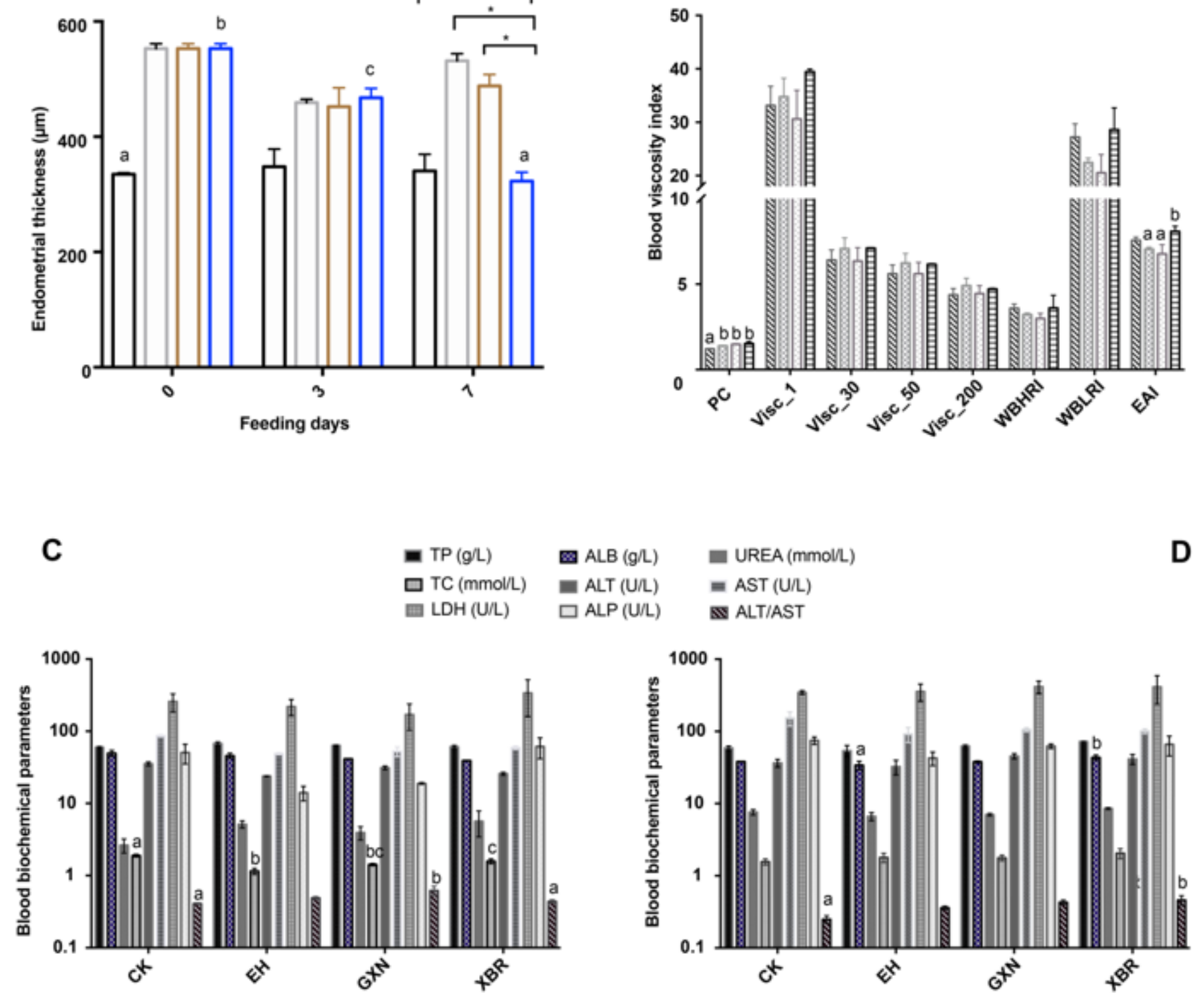

Figure 4

The effects of XBR drug on the endometrial hyperplasia (EH) and blood stasis (BS) rats. (A) The thickness of endometrium in the EH rats. (B) The levels of blood viscosity index in BS rats. (C) The alterations of blood biochemical parameters at $3 \mathrm{~d}$ gavage treatment. (D) The alterations of blood biochemical parameters at $7 \mathrm{~d}$ gavage treatment. Values are means \pm SEM. abc Different superscripts or * asterisk indicate the significant difference ( $p<0.05$, t-Student test, $n \geq 4)$. Abbreviations: PC, plasma concentration; Visc_1/30/50/200, whole blood viscosity; WBHRI, whole blood high-shearing relative index; WBLRI, whole blood low-shearing relative index; EAl, erythrocyte aggregation index. 
A

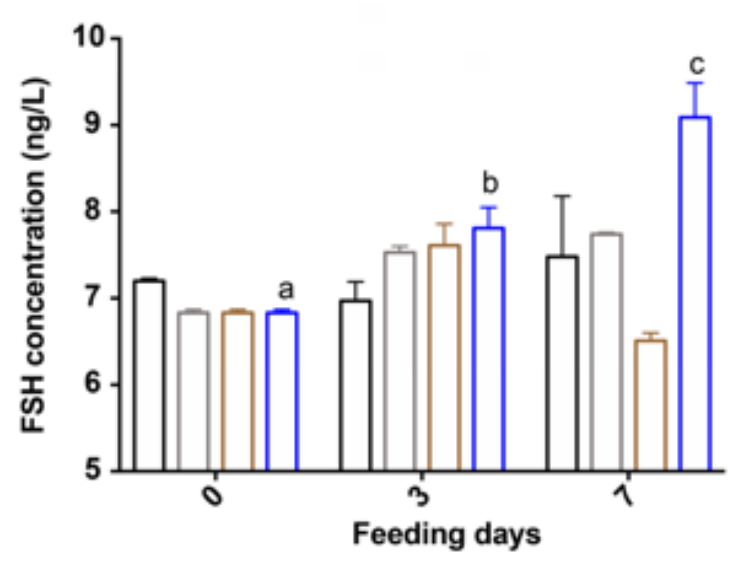

C

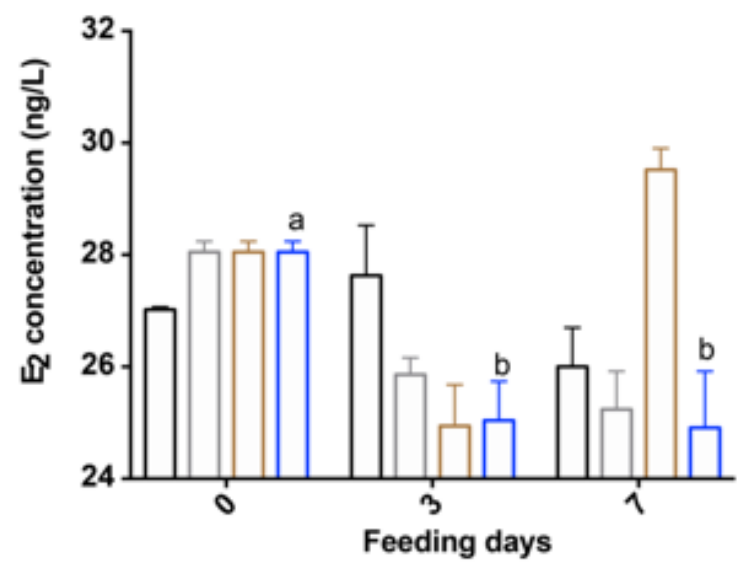

B

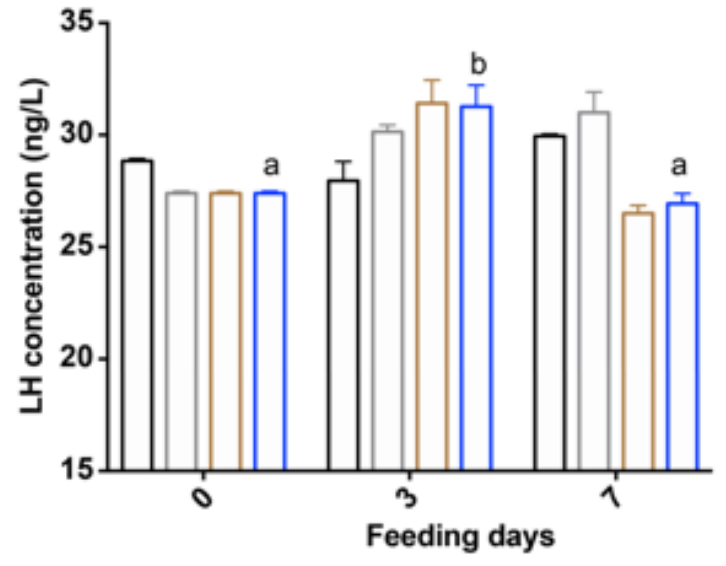

$\square \mathrm{CK}$

$\square \mathrm{EH}$

$\square$ GXN

$\square$ XBR

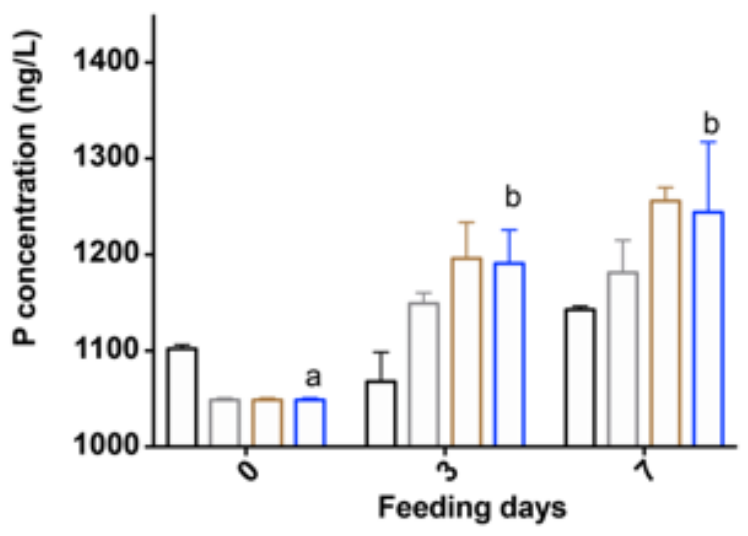

Figure 5

Effects of XBR drug on the hormone expression in the endometrial hyperplasia rats. (A) Levels of FSH in the serum of rats. (B) Levels of LH in the serum of rats. (C) E2 levels in the serum of rats. (D) P levels in the serum of rats. Values are means \pm SEM. abc Different superscripts indicate the significant difference $(p<0.05$, t-Student test, $n=4)$. 

A
$\square \mathrm{CK} \quad \square \mathrm{EH} \quad \square \mathrm{GXN} \quad \square \mathrm{XBR}$
B
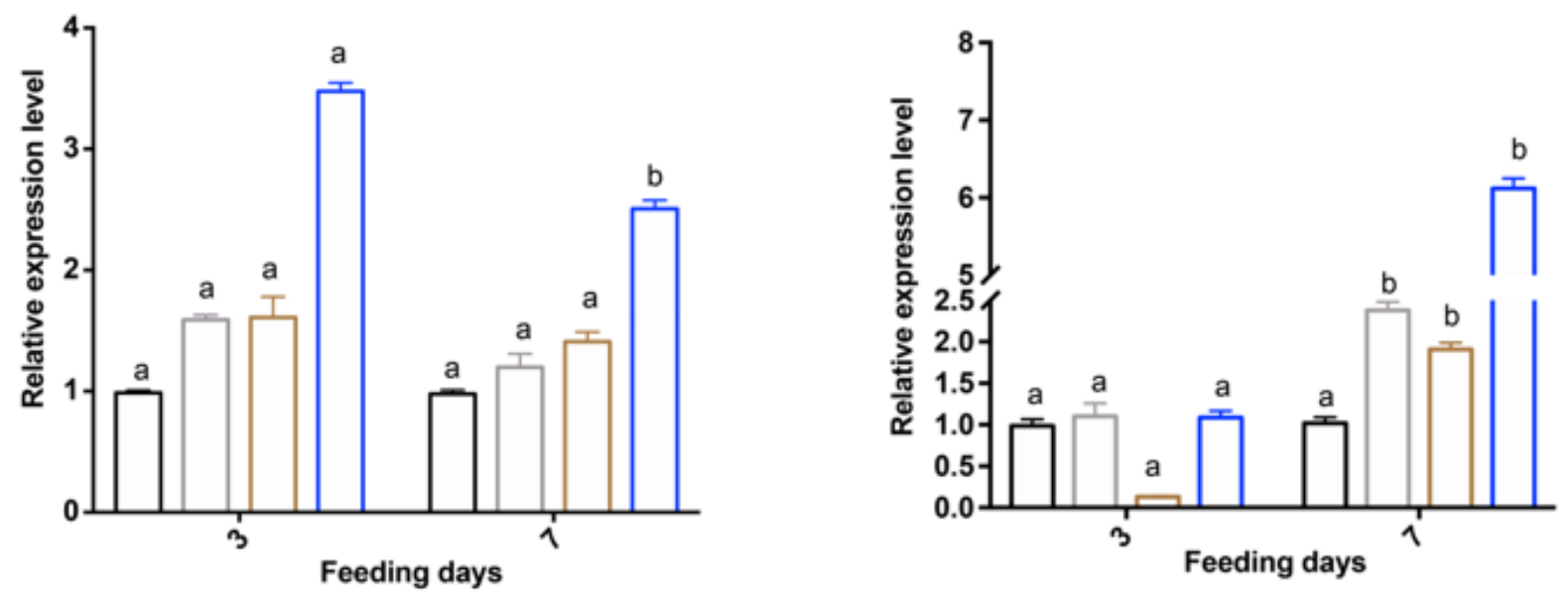

Figure 6

Effects of XBR drug on the MMP-1 and TIMP-1 expression in the endometrial hyperplasia rats. (A) mRNA expression levels of MMP-1 in endometrium (B) mRNA expression levels of TIMP-1 in endometrium . Values are means \pm SEM. ab Different superscripts indicate the significant difference $(p<0.05$, t-Student test, $n=4)$.

\section{Supplementary Files}

This is a list of supplementary files associated with this preprint. Click to download.

- Additionalfile1TableS1.docx

- Additionalfile2TableS2.docx

- Additionalfile3FigS1.docx

- Additionalfile4TableS3.docx

- Additionalfile5Figs2.docx

- Additionalfile6FigS3.docx

- Additionalfile7FigS4.docx

- Additionalfile8FigS5.docx

- Supplementarydocument.docx 